\title{
Bio synthesis and characterization of silver nanoparticles using Lagenaria siceraria leaf extract and their antibacterial activity
}

\author{
B. Anandh*, A. Muthuvel, M. Emayavaramban \\ Department of Physics, Government Arts and Sciences College, C. Mutlur, Chidambaram, India \\ *E-mail address: emayavarambanm@gmail.com
}

\begin{abstract}
The present investigation demonstrates the formation of silver nanoparticles by the reduction of the aqueous silver metal ions during exposure to the Lagenaria siceraria leaf extract. The synthesized AgNPs have characterized by UV-visible spectroscopy, X-ray diffraction (XRD) and Fourier transform infrared spectroscopy (FT-IR) techniques. AgNPs formation has screened by UV-visible spectroscopy through colour conversion due to surface plasma resonance band at $427 \mathrm{~nm}$. X-ray diffraction (XRD) confirmed that the resulting AgNPs are highly crystalline and the structure is face centered cubic (fcc). FT-IR spectrum indicates the presence of different functional groups present in the biomolecules capping the nanoparticles. Further, inhibitory activity of AgNPs and leaf extract were tested against human pathogens like gram-pastive (Staphylococcus aureus, Bacillus subtilis), gram-negative (Escherichia coli and Pseudomonas aeruginosa). The results indicated that the AgNPs showed moderate inhibitory actions against human pathogens than Lagenaria siceraria leaf extract, demonstrating its antimicrobial value against pathogenic diseases.
\end{abstract}

Keywords: Green synthesis; Silver nano particles; Lagenaria siceraria; Antibacterial Activity

\section{INTRODUCTION}

Nanotechnology, involves design, synthesis, and manipulation of structures in particles with dimension smaller than $100 \mathrm{~nm}$ for specific functions [1]. Nanoparticles are classified primarily into two types, viz organic and inorganic nanoparticles. The nanoparticles of carbon are called the organic nanoparticles. Magnetic nanoparticles, noble metal nanoparticles (platinum, gold and silver) and semiconductor nanoparticles (titanium dioxide, zinc oxide and zinc sulfide) are classified as inorganic nanoparticles [2]. Noble metal nanoparticles (NPs) have attracted a significant interest in materials and biological sciences owing to their potential applications in catalysis, biosensors, biomedical, photonics and heat transfer [3]. Silver (Ag) nanoparticles have high therapeutic potential and exhibit good antimicrobial activity. Ag nanoparticles have a wide range of antimicrobial activities and exhibit high performance even at a very low concentration. Ag nanoparticles have been identified to possess good potential for the treatment of cancer [4]. Nanoparticles play an important role in pharmaceutical, industrial and biotechnological applications. In particular, the silver nanoparticles are proved to have potential antibacterial, antifungal and antiplasmodial and larvicidal properties. The physical and chemical processes [5] are the classical general 
methods used for the fabrication of nanoparticles, but these methods are not environmentally benign [6] and due to the presence of some toxic metals in the synthesis process that may create some dicey effects in biomedical applications. Recently, utilizationof biological resources for nanoparticle synthesis has becomean alternative process for nanotechnologists.

Biological resourcesare vast, diversified and richest systems that could be employed for nanoparticles synthesis, such as bacteria, fungi and plants [7-9], with each system having its own advantages over conventional methods. Among them, plant materials have been of special interest to scientific community due to their eco-friendliness and are advantageous over other biological processes because they eliminate the elaborate process of maintaining cell structures and can also be suitably scaled up for large scale synthesis of nanoparticles. AgNPs synthesis using leaf extract has already been reported with various plants [10-14]. Reported that the banana peel extract mediated AgNPs haves hown efficient antimicrobial activity towards most of the tested fungal and bacterial cultures (Ramamurthy et al).

In the present study, we report the biosynthesis of AgNPs using the extract of Lagenaria siceraria leaf, Bottle gourd (Lagenaria siceraria (Mol.) standley, family Cucurbitaceae) commonly called as Dudhi or Ghiya is widely cultivated in the tropical and subtropical regions of the world, Lagenaria siceraria, which has diuretic and anti-swelling effects, is usedas food. A decoction of Lagenaria siceraria is employed in the treatment of anasarca, ascites and beriberi [15].

In the present investigation silver nanoparticles synthesized by biosynthesis method at room temperature using Lagenaria siceraria leaf as a capping agent. The prepared nanoparticles were characterized by various methods such as UV-visible spectroscopy, XRD, and FT-IR, analysis. The biomedical applications such as antibacterial properties of silver nanoparticles were studied by comparing with then respective salts.

\section{MATERIALS AND METHODS}

\section{1. Materials}

Silver nitrate (99.9 \%) was procured from Sigma Aldrich Chemicals, Bangalore, India. All other regents used in the reaction were of analytical grade with maximum purity. Deionized water was used throughout the experiment. The fresh leaf of Lagenaria siceraria (Figure1) was obtained from karuppur at Chidambaram, Tamil Nadu, India.

\section{2. Preparation of leaf extracts}

The fresh leaves of Lagenaria siceraria, without any infection, were collected and $50 \mathrm{~g}$ of the leaves were weighed and washed thoroughly in tap water for $10 \mathrm{~min}$ in order to remove the dust particles and rinsed briefly in deionized water before use. The leaves broth solution was prepared by taking $50 \mathrm{~g}$ of washed and finely cut leaves in a $500 \mathrm{~mL}$ Erlenmeyer flask along with $100 \mathrm{ml}$ of deionized water and then boiling the mixture at $85^{\circ} \mathrm{C}$ for $20 \mathrm{~min}$. After boiling, the solution was filtered through Whatman No.1 filter paper. The filtered extract was stored in the refrigerator for further experiments as reducing agent and stabilizer. Photograph of colloids Lagenaria siceraria leaf extract, $\mathrm{AgNO}_{3}$ metal ion Solution and pale orange color indicating the formation of AgNPs.shown in Figure 2. 


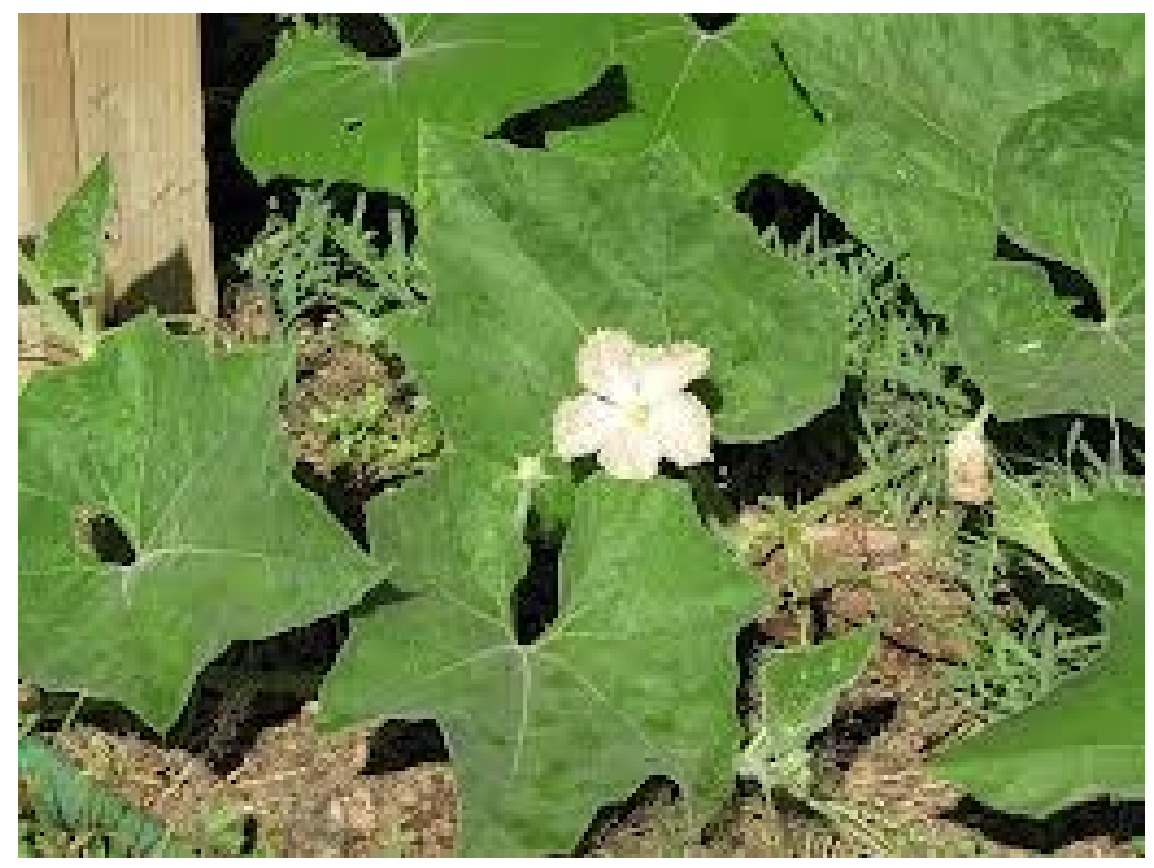

Figure 1. Photograph of Lagenariasiceraria leaf.

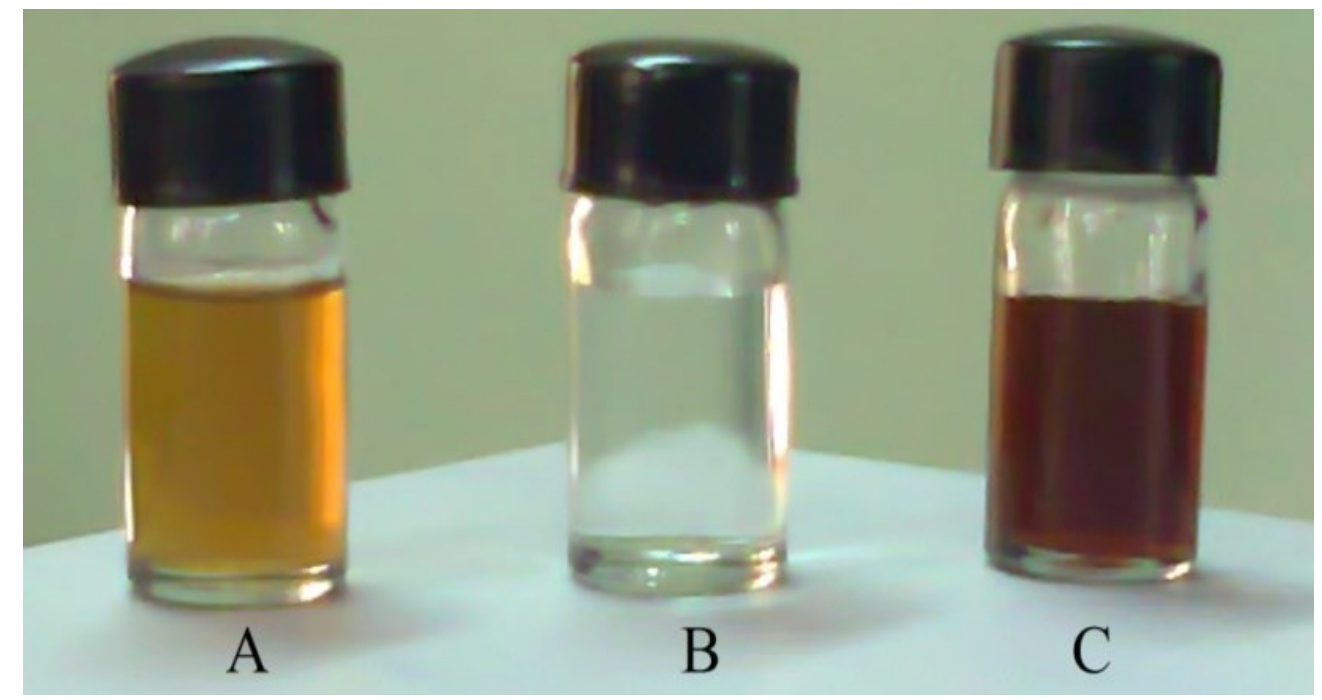

Figure 2. Photograph of colloids (A) Lagenaria siceraria leaf extract, (B) $\mathrm{AgNO}_{3}$ metal ion Solution and $(\mathrm{C})$ pale orange color indicating the formation of AgNPs.

\section{3. Biosynthesis of silver nanoparticles}

For the biosynthesis of silver nanoparticles, the leaf extract $(0.2,0.4,0.6,0.8$ and $1 \mathrm{ml})$ was added to a vigorously stirred $10 \mathrm{ml}$ of $\mathrm{AgNO}_{3}$ and kept at room temperature to get the colloids gm $_{1}-\mathrm{gm}_{5}$. For $\mathrm{gm}_{1}$ the reduction was slow and completed in $36 \mathrm{~h}$ (approximately). The reduction rate is found to increase with an increase in the quantity of the leaf extract. For $\mathrm{gm}_{5}$ fast reduction occurred as indicated by pale orange color of the solution and the reaction rate was completed within $4 \mathrm{~h}$. 


\section{4. Characterization of AgNPs.}

\section{4. 1. UV-visible spectral analysis}

The bioreduction of the $\mathrm{Ag}^{+}$ions in the solution was monitored by changes in color. Our observations indicated that the extracellular components of the Lagenaria siceraria leaf reduced the $\mathrm{Ag}^{+}$ions. The absorption spectrum of this solution was recorded using a UVvisible spectrometer (Shimadzu UV-1650) from $200 \mathrm{~nm}$ to $800 \mathrm{~nm}$ at regular intervals. The AgNPs dispersed in deionized water were subjected to ultra sonication at room temperature and their surface plasmon resonance was recorded at $427 \mathrm{~nm}$. Observation of this peak was measured continuously to determine their stability.

\section{4. 2. X-ray diffraction analysis}

To determine the crystal structure, size AgNPs, the nano powder was subjected to (XRD) analysis. X-ray analysis was done using a XRD Bruker AXS D8 Advance instrument. $\mathrm{X}$-ray generator, operated at a voltage of $40 \mathrm{kV}$ and a current of $30 \mathrm{~mA}$, wherein the sample was subjected to $\mathrm{Cu} \mathrm{K \alpha}$ radiations. The scanning $(\mathrm{K}=1.5420 \AA)$ range $(2 \theta)$ was selected from $30^{\circ}$ to $80^{\circ}$ at a $0.045^{\circ} / \mathrm{min}$ continous speed. The crystallite domain size was calculated through the Debye-Scherer's formula.

\section{4. 3. Fourier transforms infrared spectroscopy analysis}

FT-IR spectroscopic studies were carried out to find possible bio-reducing agent present in the plant leaves. The wavelength spectrum of the leaf extracts before and after the addition of the samples were mixed with $\mathrm{KBr}$ powder and pelletized after drying the spectra were recorded using. Avatar 330 FT-IR spectrometer in the range of $4000-500 \mathrm{~cm}^{-1}$ at a resolution of $4 \mathrm{~cm}^{-1}$.

\section{4. 4. Antibacterial activity}

The antibacterial potential of biosynthesized AuNPs and Lagenaria sicerariawere tested against gram-positive (Staphylococcus aureus and Bacillus subtilis) and gram-negative (Escherichia coli and Pseudomonas aeruginosa) bacteria by using the agar well diffusion assay method (Perez et al., 1990). Approximately $20 \mathrm{ml}$ of molten and cooled nutrient agar media was poured into sterilised petri dishes. The plates were left overnight at room temperature to allow any contamination to appear. The discs wereplaced on Muller Hinton agar plates inoculated with each of thepreviously mentioned microorganisms. $25 \mu \mathrm{L}$ of ciprofloxacin was used as positive control (PC). The test plates were incubated at $37{ }^{\circ} \mathrm{C}$ for $24 \mathrm{~h}$. After the incubation period, the zone of inhibition (in mm diameter) was observed and tabulated.

\section{RESULTS AND DISCUSSION}

\section{1. UV-Vis spectral analysis}

$\mathrm{UV}-\mathrm{Vis}$ spectroscopy is one of the most important techniques to characterize the metal nanoparticles. The absorption behavior arises due to surface Plasmon resonance (SPR), which originates from coherent oscillations of electrons in the conduction band of nanoparticles induced by the electromagnetic field. The SPR phenomenon arises when nanoparticles are irradiated with visible light, because of the collective oscillations of the conduction electrons. 
It is well known that AgNPs exhibit a pale orange color in aqueous solution due to the excitation in UV-visible spectrum depending upon the particle size [16]. Figure (3), shows the UV-visible spectra of the silver colloids $\left(\mathrm{gm}_{1}-\mathrm{gm}_{5}\right)$.

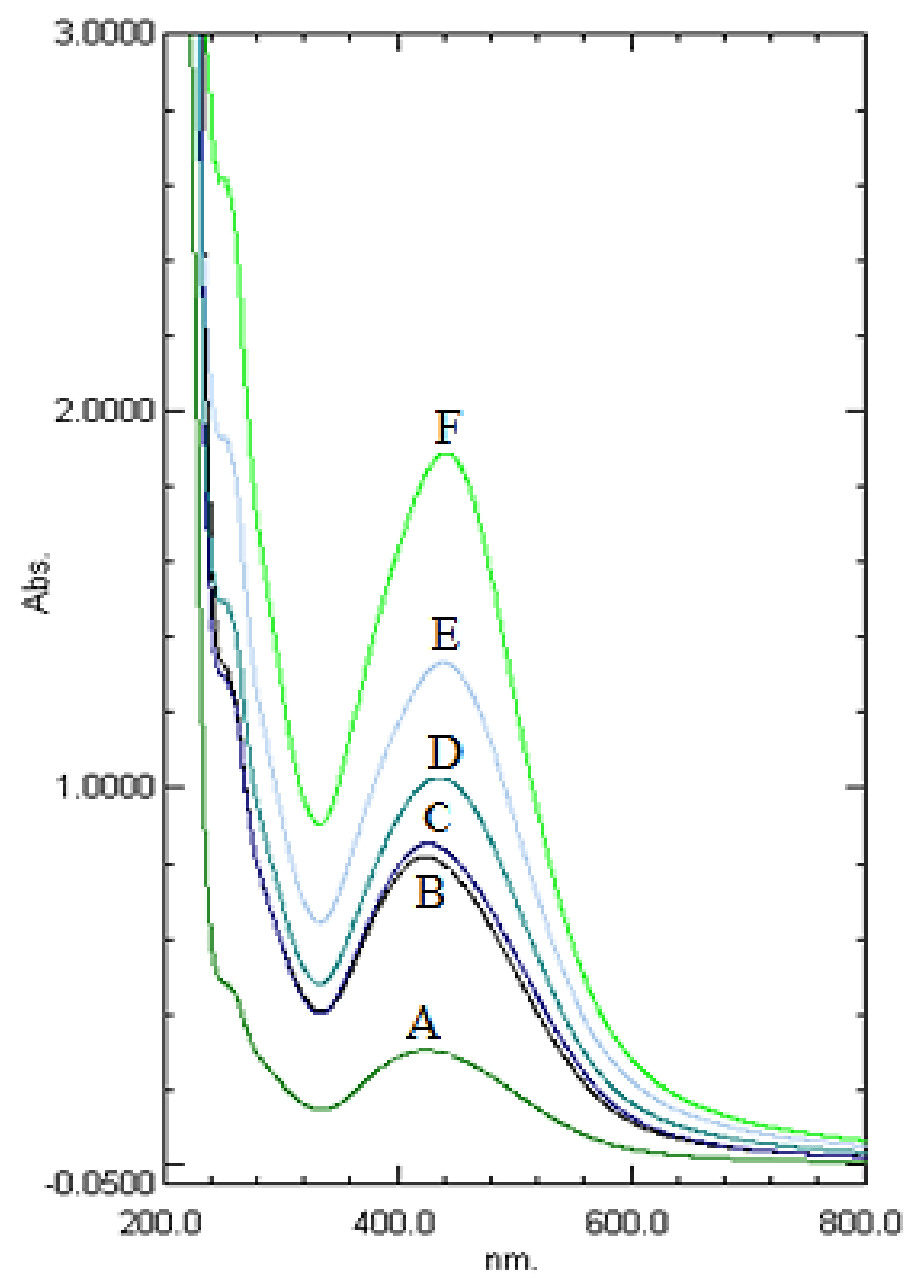

Figure 3. UV-Vis spectra of AgNPs prepared at different leaf extract concentration (gm1) $0.2 \mathrm{~mL},(\mathrm{gm} 2) 0.4 \mathrm{~mL},(\mathrm{gm} 3), 0.6 \mathrm{~mL},(\mathrm{gm} 4), 0.8 \mathrm{~mL}$ and $(\mathrm{gm} 5) 1 \mathrm{~mL}$.

The scale of wavelength was fixed between 200 to $800 \mathrm{~nm}$, the surface plasmon resonance (SPR) of the AgNPs obtained at $427 \mathrm{~nm}$ and there was an increase in intensity till $10 \mathrm{~min}$ as a function of time without any shift in the peak wavelength (Fig. 3). It suggested that the reduction of silver ions reaches saturation within $10 \mathrm{~min}$ of reaction, and after that, only slight variations can be noted in the intensity of SPR bands and moreover the reaction is completed in $10 \mathrm{~min}$. From the results of UV-visible spectra the gradual color change from pale orange color was observed during reaction with varying concentration of lagenaria scieraria leaf extract which are characteristics of the SPR of different size of AgNPs in solution. Initially, the maximal SPR peaks of AgNPs showed broaden in bandwidth of the peak with the decreased concentration of leaf extract $\left[\mathrm{gm}_{1}(0.2 \mathrm{~mL})\right.$ and $\left.\mathrm{gm}_{2}(0.4 \mathrm{ml})\right]$. At higher concentrations, the SPR peak is shifted towards shorter wave length region which shows a decrease in particle size [17]. 
The size and shape of the AgNPs synthesized using the leaf extract of lagenaria scieraria were found to be very sensitive to the quantity of the extract and the amount of extract is increased, the stronger the interaction between the extract biomolecules and nascent nanoparticles.

\section{2. X-ray analysis}

The structure of biologically synthesized AgNPs was analysed by XRD measurements. A typical XRD pattern of the AgNPs synthesized using lagenaria scieraria leaf extract was found to possess a fcc (JCPDS no. 04-0784) structure as shown in Figure (4). The Bragg reflections at $2 \theta=38.17$, and 44.38 , can be indexed to the (llll 111$)$ and $\left(\begin{array}{lll}2 & 0 & 0\end{array}\right)$, orientations, respectively, confirmed the presence of AgNPs. These results clearly indicated that the AgNPs were composed of highly crystalline nano structures [18]. The mean size of silver nanoparticles was calculated using the Debye-Scherer's equation by determining the width of the (111) Braggs reflection. The size of the nanoparticles was thus determined to be about 42 nm for AgNPs.

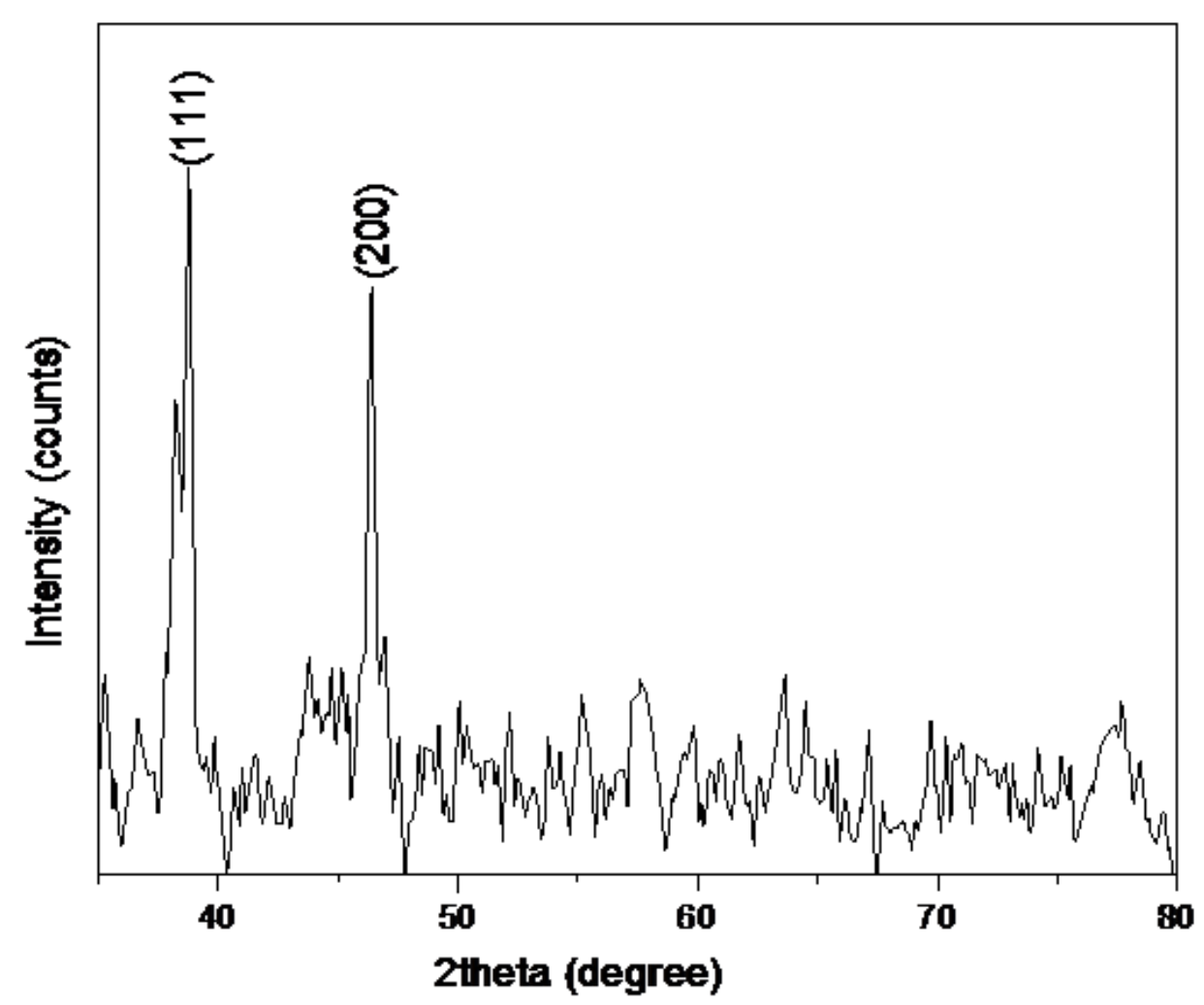

Figure 4. XRD pattern of dried powder of biosynthesized AgNPs.

\section{3. Fourier transforms infrared (FT-IR) specrtcopy analysis}

FT-IR measurement was carried out to identify the possible biomolecules in lagenaria scieraria leaf responsible for capping leading to efficient stabilization of the AgNPs. FT-IR spectroscopy is used to identify the functional groups that play an important role in reducing and stabilizing the AgNPs prepared using lagenaria scieraria leaf extract. The FT-IR spectra 
of lagenaria scieraria leaf extract and AgNPs is shown in Figure (5 ( $a$ and b)) respectively. The IR spectra of lagenaria scieraria leaf extract shows prominent bands at 3396, 1731, 1626, 1463, 1089 and $1033 \mathrm{~cm}^{-1}$. The strong band observed at $3396 \mathrm{~cm}^{-1}$ corresponds to hydroxyl functional groups in polyphenols [19]. The IR band due to $\mathrm{C}=\mathrm{O}$ stretch is observed at $1731 \mathrm{~cm}^{-1}$. The band observed at $1626 \mathrm{~cm}^{-1}$ is identified as the amide I and arises due to the carbonyl stretch vibrations in the amide linkages of the proteins. The other band at $1463 \mathrm{~cm}^{-}$ ${ }^{1}$ is assigned to the methylene scissoring vibrations of proteins. The peaks at $1089 \mathrm{~cm}^{-1}$ and $1033 \mathrm{~cm}^{-1}$ are the characterstics of C-O stretch of carboxylic acids and ether and alcoholic groups [20]. It is found that a similar pattern with a slight shift in wave numbers is repeated for FT-IR spectra of functionalized AgNPs at 3417, 1749, 1616, 1099 and $1022 \mathrm{~cm}^{-1}$. A noticeable change observed on comparing the IR spectra of lagenaria scieraria leaf extract and functionalized AgNPs is that the relative intensity of band at 3417, 1731, 1616, 1099 and $1022 \mathrm{~cm}^{-1}$ decreased considerably suggesting the predominant involvement of water soluble compounds such as flavonoids and terpenoids in reduction of $\mathrm{Ag}^{+}$ions.

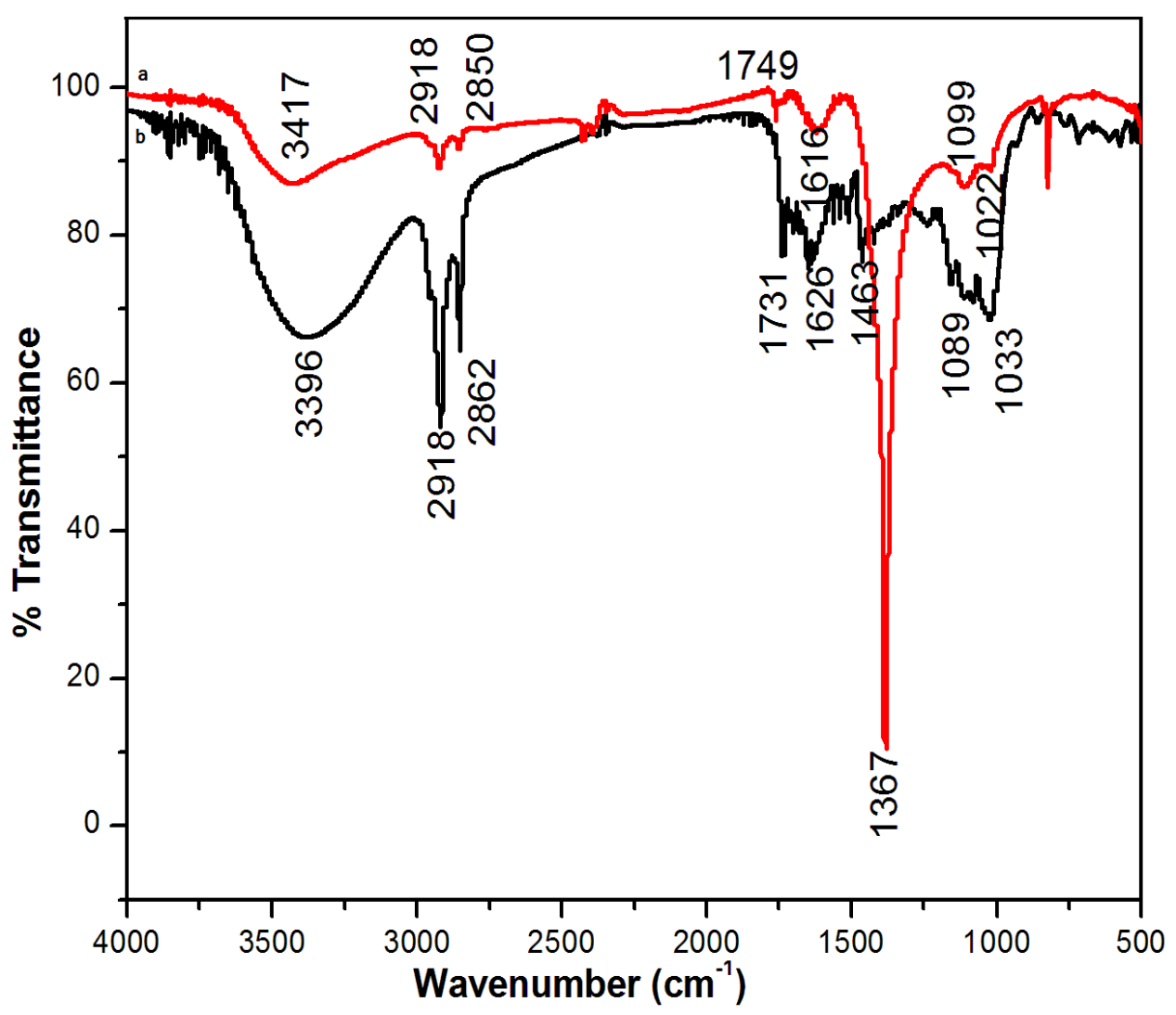

Figure 5. FTIR spectra of dried powder of AgNPs (a) and (b) dried powder of Lagenaria siceraria leaf extract.

The phytochemical analysis of the dried leaf extract of lagenaria scieraria has been reported to show the presence of vitamins, carotenoids, terpenoids, alkaloids, flavonoids, lignans and phenolics. The flavonoids present in the leaf extract are powerful reducing agents which may be responsible for the reduction of $\mathrm{Ag}^{+}$ions. The flavonoids and terpenoids present in lagenaria scieraria leaf extract can act as surfactant to attach on the surface of 
AgNPs and it stabilizes AgNPs through electrostatic stabilization. Thus it is found that lagenaria scieraria leaf extract has the ability to perform dual functions of reduction and stabilization of AgNPs.

\section{4. Antibacterial activity}

Silver nanoparticles pretense to have strong bactericidal activity against gramnegative and gram-positive bacteria including multidrug resistant strains. In the present study antibacterial activity of the biosynthesized AgNPs against different human pathogens is shown in Figure (6). It is apparent that the AgNPs showed inhibition zone against almost all the test organisms (Table 1). The synthesized AgNPs were found to have higher inhibitory action when compared to the Lagenaria siceraria. Moreover, AgNPs exhibit effective zone of inhibition against gram-negative bacteria ( $E$. coli and $P$. aeruginosa) compared to the gram-positive bacteria (S. aureus and B. subtilis).

Table 1. Inhibitory action of control, Lagenaria siceraria leaf extract and AgNPs against human Pathogenic bacteria.

\begin{tabular}{|c|c|c|c|c|}
\hline \multirow{2}{*}{ Plate } & Culture & \multirow{2}{*}{ Standard } & \multicolumn{2}{|c|}{ Concentration $(\mu \mathrm{l})$} \\
\cline { 4 - 5 } & & & $100 \mu 1$ leaf & $100 \mu \mathrm{l}$ AgNPs \\
\hline 1 & Staphylococcus saprophyticus & 25 & 15 & 21 \\
\hline 2 & Bacillus subtilis & 25 & 13 & 18 \\
\hline 3 & Escherichia coli & 29 & 16 & 21 \\
\hline 4 & Pseudomonas aeruginosa & 26 & 15 & 20 \\
\hline
\end{tabular}

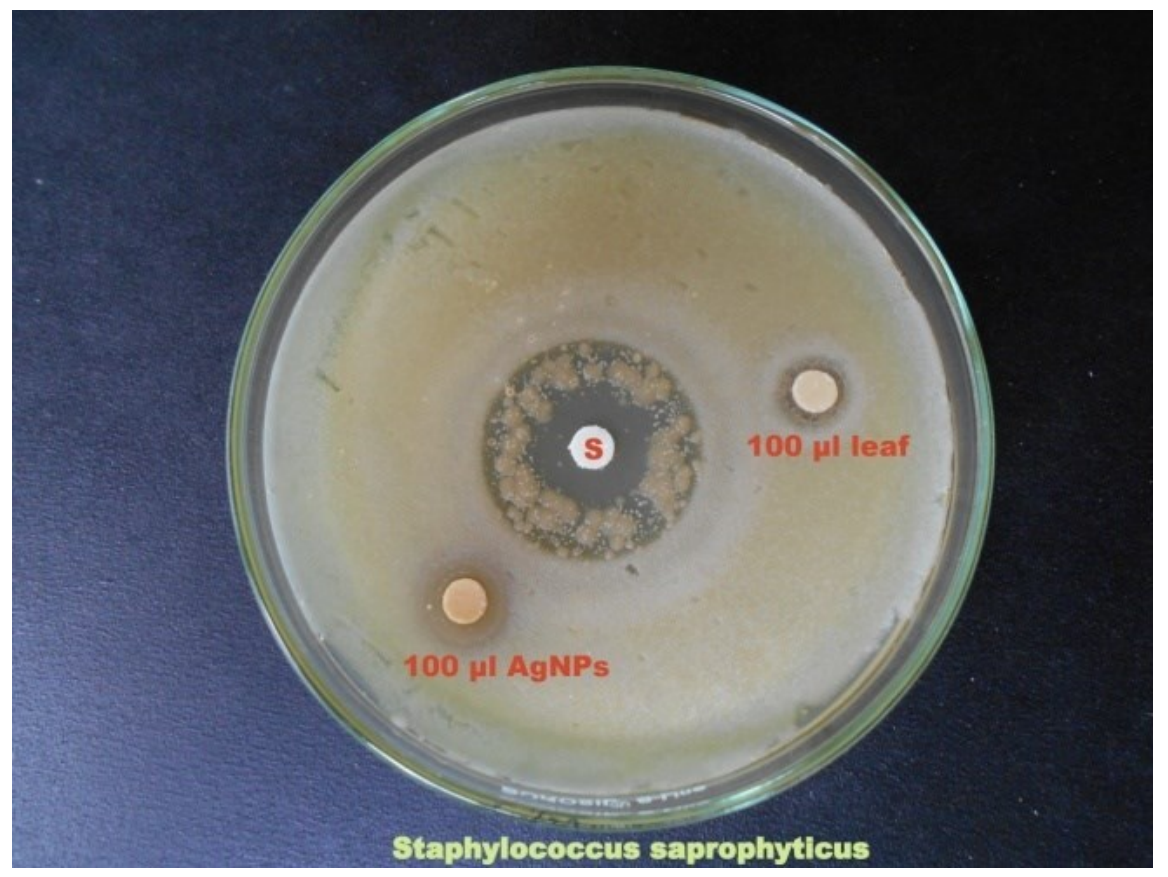



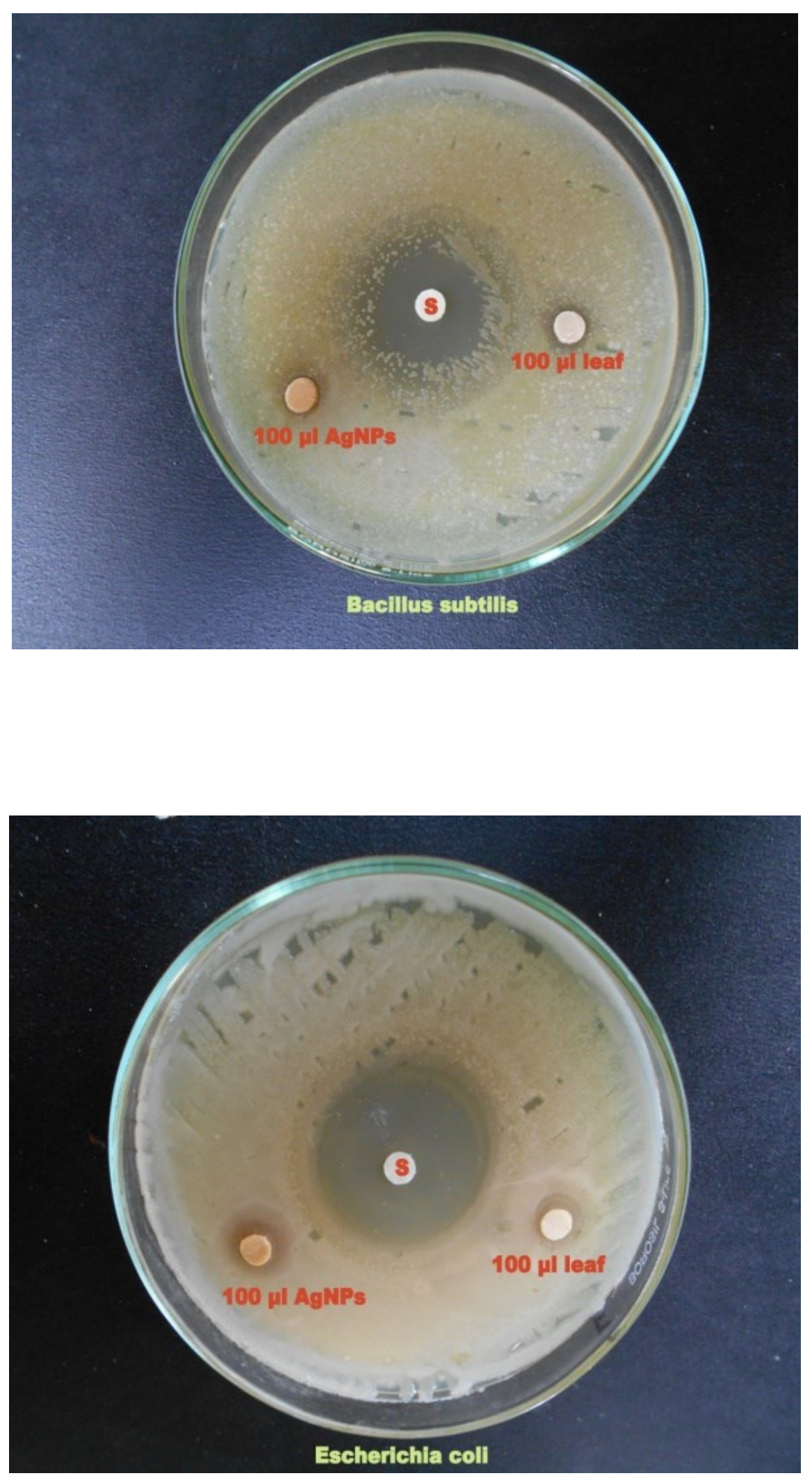


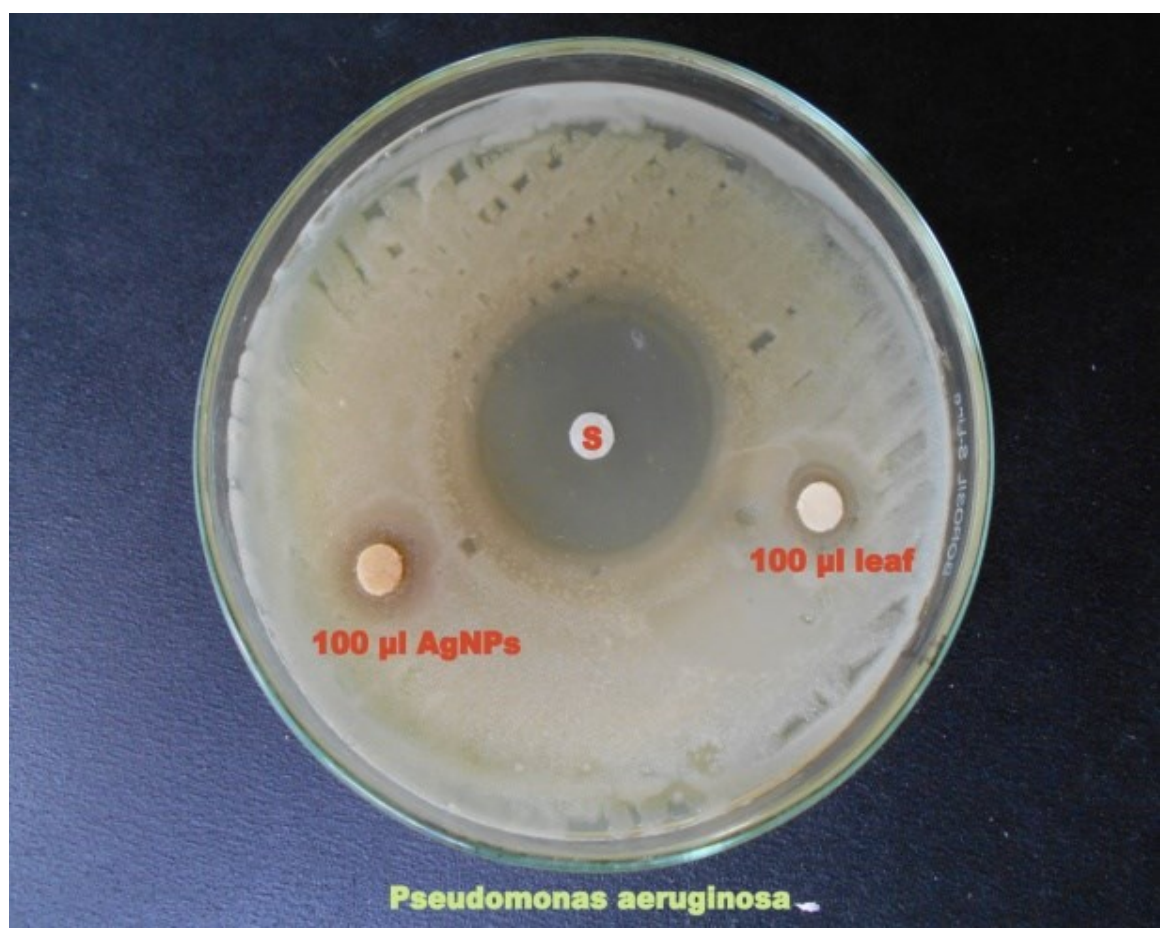

Antibacterial effects of AgNPs obeyed an action mechanism of antibacterialactivity. The potential reason for the antibacterial activity of silver is that AgNPs may attach to the surface of the cell membrane disturbing permeability and respiration functions of the cell. Smaller AgNPs having the large surface area available for interaction would give more antibacterial effect than the larger AgNPs. It is also possible that AgNPs not only interact with the surface of membrane, but can also penetrate inside the bacteria.

\section{CONCLUSION}

It has been predicted that the leaf extract of Lagenaria siceraria leaf is capable of producing AgNPs extracellularly and these nanoparticles are quite stable in solution due to capping likely by the flavonoids and terpenoids present in the leaf extract. This is an efficient, eco-friendly and simple process. The AgNPs showed potential antibacterial activity against some human pathogens. Therefore, nanoparticles of silver in combination with commercially available antibiotics could be used as an antimicrobial agent after further trials on experimental animals.

\section{References}

[1] G. Sathishkumar, C. Gobinath, K. Karpagam, V. Hemamalini, K. Premkumar, S. Sivaramakrishnan, Colloids and Surfaces B: Biointerfaces 95 (2012) 235-240.

[2] N. Asmathunisha, K. Kathiresan, Colloids and Surfaces B: Biointerfaces 103 (2013) 283-287. 
[3] AM. Md Jani, D. Losic, N.H. Voelcker. Progress in Materials Science 58 (2013) 636704.

[4] S. Govindan, E. A. K. Nivethaa, R. Saravanan, V. Narayanan, A. Stephen, Applied Nanoscience 2 (2012) 299-303.

[5] M. Gnanadesigan, M. Anand, S. Ravikumar, M. Maruthupandy, M. Syed Ali, V. Vijayakumar, A. K. Kumaraguru, Applied Nanoscience 2 (2012) 143-147.

[6] V. Ganesan, A. Astalakshmi, P. NimaC. Arunkumar. International Journal of Current Science 6 (2013) 87-93.

[7] Z. Ranjbar Navazi, M. Pazouki, F.SadatHalek. Iranian journal of biotechnology 8 (2010) 1-8.

[8] K.C. Bhainsa, S.F. D’Souza, Journal of Colloids and Surfaces B 47 (2006) 160.

[9] S. Iravani, Green Chemistry 13 (2011) 2638.

[10] SS. Shankar, A. Rai, A. Ahmad, M. Sastry, Journal Colloid Interface Sciences 275 (2004) 496-502.

[11] V. Kathiravan, S. Ravi, S. Ashokkumar, Spectrochimica Acta Part A: Molecular and Biomolecular Spectroscopy 130 (2014) 116-121.

[12] S. Ashokkumar, S. Ravi, S. Velmurugan, Spectrochimica Acta Part A: Molecular and Biomolecular Spectroscopy 115 (2013) 388-392.

[13] Abduz Zahir, A. Abdul Rahuman, Veterinary Parasitology 187 (2012) 511-520.

[14] G. Sathishkumar, C. Gobinath, K. Karpagam, V. Hemamalini, K. Premkumar, S. Sivaramakrishnan, Colloids and Surfaces B: Biointerfaces 95 (2012) 235-240.

[15] H.X. Wang, T.B. Ng,Life Sciences 67 (2000) 2631-2638.

[16] S. Samanta, P. Sarkar, S. Pyne, G. Prasad Sahoo, A. Misra, Journal of Molecular Liquids 165 (2012) 21-26.

[17] Liu, C. Chris Mi, S. Member, B.Q. Li. Ieee, Transactions on Nanobioscience 7 (2008) 3-9.

[18] S. Khosravi-Gandomani, R.Yousefi, F.Jamali-Sheinic, N. Ming Huang. Ceramics International 40 (2014) 7957-7963.

[19] D.S. Sheny, J. Mathew and D. Philip. Spectrochimica Acta Part A: Molecular and Biomolecular Spectroscopy 79 (2011) 254-260.

[20] A.J. Kora, R. B. Sashidhar and J. Arunachalam, Carbohydrate Polymers 82 (2010) 670675. 